\title{
FLORÍSTICA E ESTRUTURA DA COMUNIDADE ARBÓREA DE DUAS ÁREAS DE CERRADO SENTIDO RESTRITO NO NORTE DE MINAS GERAIS
}

\author{
Fernanda Vieira da Costa $^{1}$, Karla Nunes Oliveira ${ }^{1}$, Yule Roberta Ferreira Nunes ${ }^{2}$, Gisele Cristina de Oliveira Menino ${ }^{1}$, \\ Diego Oliveira Brandão ${ }^{3}$, Lucimar Soares de Araújo ${ }^{4}$, Weslane Oliveira Miranda ${ }^{4}$, Santos D`Ângelo Neto ${ }^{5}$
}

(recebido: 5 de novembro de 2008; aceito: 30 de abril de 2010)

\begin{abstract}
RESUMO: Este estudo teve como objetivo comparar os aspectos florísticos e fitossociológicos de duas áreas próximas de Cerrado Sentido Restrito em Grão Mogol, norte de Minas Gerais. As comunidades arbóreas foram avaliadas por meio do método de pontos quadrantes. Em cada área, foram distribuídos pontos equidistantes $10 \mathrm{~m}$ entre si ao longo de seis transectos de $70 \mathrm{~m}$, totalizando 48 pontos por área. Foram medidas as distâncias até o ponto central, a altura e o CAP das quatro plantas correspondentes aos quadrantes de cada ponto. Foram considerados os indivíduos arbóreos vivos com DAP $\geq 3 \mathrm{~cm}$ e altura $\geq 1,5 \mathrm{~m}$. Amostrou-se um total de 73 espécies nas duas áreas, sendo 48 na área 1 e 54 na área 2. As famílias com maior representatividade foram Fabaceae e Vochysiaceae. A densidade total estimada para a área 1 foi de 1275,51 ind. ha ${ }^{-1}$ e 1580,58 ind. ha ${ }^{-1}$ para a área 2. Em ambas as áreas, Qualea parviflora e Eriotheca pubescens apresentaram o maior valor de importância (IVI). Os valores de diversidade $\left(H^{\prime}\right)$ e de equabilidade $\left(J^{\prime}\right)$ foram de 3,13 e 0,87 , e de 3,27 e 0,84 , respectivamente, para a área 1 e 2 . O índice de similaridade de Sorensen entre as áreas foi de 0,68. A distribuição diamétrica e de altura das comunidades estudadas apresentaram forma de J-invertido, indicando um balanço positivo entre recrutamento e mortalidade, resultante do processo de sucessão secundária. As áreas estudadas apresentam o padrão de riqueza de espécies lenhosas do Cerrado Sentido Restrito, entretanto, a área 2 apresentou valores florísticos e estruturais mais elevados, indicando estágio sucessional mais avançado.
\end{abstract}

Palavras-chave: Diversidade, fitossociologia, método de quadrantes, similaridade florística.

\section{FLORISTIC COMPOSITION AND WOOD COMMUNITY STRUCTURE OF TWO CERRADO STRICTO SENSU AREAS ON NORTH OF MINAS GERAIS}

\begin{abstract}
This study aimed to compare floristic composition and the phytosociological structure between two fragments of stricto sensu cerrado in Grão Mogol, North of Minas Gerais, Brazil. The tree communities were evaluated using the quarter point method. In each fragment, points were distributed equidistant $10 \mathrm{~m}$ among them along six transects of $70 \mathrm{~m}$, totaling 48 points per fragment. It was measured, in each point, the height, CBH of four plants to the corresponding quarters in relation to central point. It was considered the individual trees sampled with $\mathrm{DHB} \geq 3 \mathrm{~cm}$ and height $\geq 1.5 \mathrm{~m}$. It was sampled a total of 73 species in the two fragments, being 48 species in fragment 1, distributed in 41 genera and 24 families, while 54 species were found in fragment 2, distributed in 47 genera and 28 families. The most representative families were Fabaceae and Vochysiaceae. The total density estimated for the fragment 1 was 1275.51 ind. $\mathrm{ha}^{-1}$ and $1580.58 \mathrm{ind}$. ha $\mathrm{a}^{-1}$ to the fragment 2. In both fragments, Qualea parviflora and Eriotheca pubescens showed the highest importance value (IVI). The diversity $\left(H^{\prime}\right)$ and equability $(J)$ values were 3.13 and 0.87 for fragment 1 , and 3.27 and 0.84 for fragment 2, respectively. The Sorensen's similarity index was 0.68 . The diameter and height distribution of the study communities presented inverted-J form, indicating a positive balance between recruitment and mortality, coming from the secondary sucessional process of the communities. The study areas showed woody species richness pattern of the sensu stricto cerrado, however, the area 2 showed higher floristic and structural values, indicating more advanced sucessional stage.
\end{abstract}

Key words: Diversity, phytossociology, quarter's point method, floristic similarity.

\section{INTRODUÇÃO}

Inicialmente, o Cerrado compreendia uma área de aproximadamente dois milhões de $\mathrm{km}^{2}$ do território brasileiro, sendo considerado o segundo maior bioma do país (EITEN, 1993). Entretanto, a cobertura original do Cerrado foi reduzida em mais de $37 \%$, em razão das ocupações humanas desordenadas, a exploração irracional

\footnotetext{
${ }^{1}$ Bióloga, Mestranda em Ciências Biológicas - Departamento de Biologia Geral - Centro de Ciências Biológicas e da Saúde - Universidade Estadual de Montes Claros - Cx.P. 126 - 39401-089 - Montes Claros, MG - fecostabio@gmail.com, karlanunesbio@gmail.com, giselecristina.biologa@yahoo.com.br

${ }^{2}$ Bióloga, Professora Dra. em Engenharia Florestal - Laboratório de Ecologia e Propagação Vegetal - Departamento de Biologia Geral Universidade Estadual de Montes Claros - Cx.P. 126 - 30401-089 - Montes Claros, MG - yule.nunes@unimontes.br

${ }^{3}$ Graduando em Ciências Biológicas - Departamento de Biologia Geral - Universidade Estadual de Montes Claros - Cx.P. 126 - $30401-089$ Montes Claros, MG - dbrandão13@gmail.com

${ }^{4}$ Graduada em Ciências Biológicas - Departamento de Biologia Geral - Universidade Estadual de Montes Claros - Cx.P. 126 - $30401-089$ Montes Claros, MG - lucimar.araujo@gmail.com, lanebio10@hotmail.com

${ }^{5}$ Agrônomo, Professor Mestre em Engenharia Florestal - Departamento de Biologia Geral - Centro de Ciências Biológicas e da Saúde Universidade Estadual de Montes Claros - Cx.P. 126 - 39401-089 - Montes Claros, MG - santosdangelo@bol.com.br
}

Cerne, Lavras, v. 16, n. 3, p. 267-281, jul./set. 2010 
dos recursos, a expansão agropecuária e o uso indiscriminado do fogo (FELFILI et al., 2002).

O Cerrado, segundo Coutinho (1978), é caracterizado por cinco fitofisionomias denominadas campo limpo, campo sujo, campo cerrado, cerrado sentido restrito e cerradão. O Cerrado Sentido Restrito ocupa cerca de $1.400 .000 \mathrm{~km}^{2}(70 \%)$ da área total do bioma e seu estrato arbóreo possui cobertura vegetal de 10 a $60 \%$ (FELFILI \& FELFILI, 2001). É considerado uma formação savânica, caracterizada pela presença de árvores baixas, inclinadas, tortuosas e, geralmente, com evidência de queimadas. A profundidade do lençol freático, a frequência de queimadas e os fatores antrópicos têm nítida influência na distribuição das suas espécies arbóreas (RIBEIRO \& WALTER, 1998). Segundo Ribeiro \& Walter (1998), por exemplo, o Cerrado Sentido Restrito Típico apresenta árvores com altura média ente 3 e $6 \mathrm{~m}$, com cobertura arbórea de 20 a 50\%. A diversidade (por meio do índice de Shannon) varia em torno de 3,0 (CARVALHO \& MARQUES-ALVES, 2008).

Por muito tempo, o Cerrado foi classificado como de baixa prioridade para conservação, principalmente por esta fisionomia ser representada por árvores com ramos tortos e o porte relativamente baixo. Entretanto, a partir de 1980, iniciou-se um esforço de pesquisa que demonstrou sua grande biodiversidade, incluindo vários endemismos (PRIMACK \& RODRIGUES, 2001). Uma recente classificação, aponta 34 biomas do planeta como áreas prioritárias para conservação (biodiversity hotspots), baseada na elevada diversidade numérica de espécies, altos níveis de endemismos e grandes taxas de destruição de habitat (CONSERVAÇÃO INTERNACIONAL DO BRASIL CI, 2009). Por atender a estes critérios, o Cerrado brasileiro foi classificado como um hotspot da biodiversidade.

Nesse sentido, o conhecimento da organização estrutural das populações de espécies arbustivo-arbóreas do Cerrado, por meio de estudos florísticos e fitossociológicos, é importante para o estabelecimento de áreas prioritárias para conservação (FELFILI et al., 2002) e para a elaboração de propostas de recuperação de áreas que sofreram distúrbios (FIEDLER et al., 2004). Apesar de vários estudos florísticos e fitossociológicos apresentarem bom nível de conhecimento vegetacional do Cerrado, a maioria é restrita à região central do Brasil, principalmente nos Estados de Mato Grosso e Goiás (FELFILI, 1997; FELFILI et al., 1993, 1997, 2002, 2007). Além disso, a grande heterogeneidade vegetacional do Cerrado, determinada pela latitude e diferentes aspectos edáficos, sofre grande interferência da ação antrópica (queimadas, corte seletivo, pastejo de animais domesticados, introdução de espécies exóticas, entre outros), causando a formação de fragmentos e áreas em diferentes estágios de sucessão (CARVALHO \& MARQUES-ALVES, 2008; RIBEIRO \& WALTER, 1998). Desse modo, o conhecimento sobre a distribuição e organização das comunidades no Cerrado ainda é escasso (ASSUNÇÃO \& FELFILI, 2004), sendo que esse entendimento se torna essencial para programas de manejo e conservação deste bioma (FELFILI \& SILVA-JÚNIOR, 2001).

A distribuição das espécies, refletida na grande diversidade de mosaicos, contribui para esta falta de informações concisas sobre o Cerrado, especificamente em Minas Gerais, onde poucos estudos foram realizados (NERI et al., 2007b) e, especialmente, na região norte do Estado, onde estes estudos são inexistentes. Dessa forma, neste trabalho, objetivou-se comparar os aspectos florísticos e estruturais das comunidades arbóreas em duas áreas próximas de Cerrado Sentido Restrito, localizadas no município de Grão Mogol, norte de Minas Gerais. Assim, procurou-se testar a hipótese de que apesar da proximidade das áreas, possíveis históricos de perturbação diferentes são determinantes da ocorrência de padrões florísticos e fitossociológios dissimilares entre as mesmas. Além disso, este estudo visa a contribuir para o conhecimento dessa vegetação na região e, consequentemente, para propostas de conservação dos remanescentes de Cerrado no norte de Minas.

\section{MATERIAL E MÉTODOS}

\section{1 Área de estudo}

A área de estudo compreende dois ambientes de Cerrado Sentido Restrito localizadas na Área de Preservação da Usina Hidrelétrica de Santa Marta (S $16^{\circ}$ 37' 44" e W 43 18' 21"') da Companhia Energética de Minas Gerais (CEMIG), em Grão Mogol, norte de Minas Gerais (Figura 1). Fisionomicamente, a região está incluída na transição entre os domínios do Cerrado e da Caatinga (RIZZINI, 1997), apresentando como principais fitofisionomias Cerrado Sentido Restrito, mata ciliar e campos rupestres. O clima da região é do tipo semiárido, com estações secas e chuvosas bem definidas. A temperatura média anual varia entre $21^{\circ}$ a $24^{\circ} \mathrm{C}$ e a precipitação média varia de 900 a $1200 \mathrm{~mm}$, com chuvas concentradas principalmente nos meses de novembro a janeiro (Instituto Nacional de Meteorologia - INMET, 2008). Quanto ao tipo de solo, predominam os latossolos, embora também ocorram solos podzólicos com textura média ou arenosa (NAIME, 1994). 


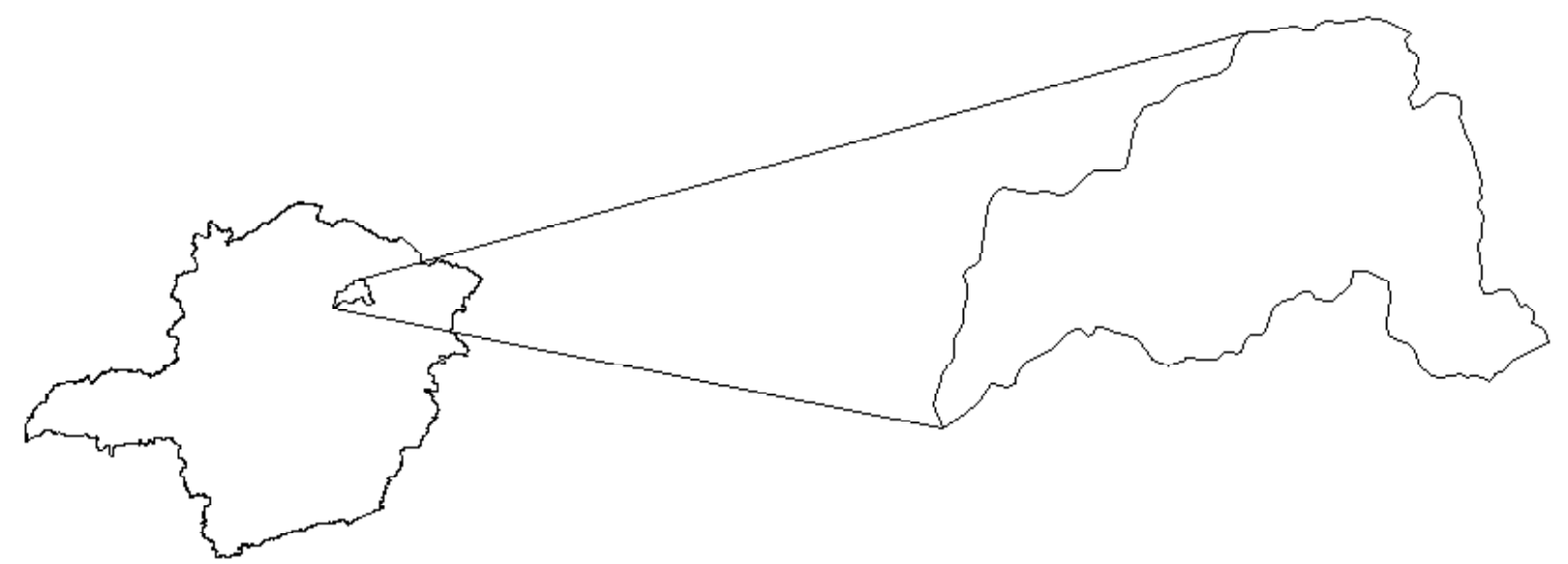

Figura 1 - Localização do município de Grão Mogol no Estado de Minas Gerais, onde fica a Área de Preservação da Usina Hidrelétrica de Santa Marta (CEMIG), MG.

Figure 1 - Location of Grão Mogol city on the Minas Gerais State, where is the Santa Marta Hydroelectric Preservation Area (CEMIG), $M G$.

As áreas estudadas se encontram em diferentes níveis de regeneração e, segundo moradores locais, a área 1 (790 m de altitude) possui histórico de perturbação recente, com sinais evidentes de pressão antrópica, por meio de corte seletivo, queimadas e conversão em pastagens. A área 2 (880 m de altitude) também é contornada por pastagens, porém encontra-se, visualmente, pelo maior porte das árvores e cobertura vegetal mais densa, em um nível de regeneração natural mais avançado. A distância entre as áreas estudadas é de aproximadamente $1 \mathrm{Km}$.

\subsection{Amostragem da vegetação}

O levantamento da estrutura e composição florística da comunidade arbórea foi realizado em junho de 2007, adotando-se o método de pontos quadrantes (COTTAM \& CURTIS, 1956), tendo em vista a facilidade e rapidez de sua execução (DURIGAN, 2004). Em cada área, os pontos foram distribuídos equidistantes $10 \mathrm{~m}$ entre si ao longo de seis transectos de $70 \mathrm{~m}$, totalizando 48 pontos por fragmento. As quatro plantas, correspondentes aos quatro quadrantes de cada ponto, com DAP (diâmetro a 1,30 m do solo) $\geq 3 \mathrm{~cm}$ e altura $\geq 1,5 \mathrm{~m}$ foram amostradas, totalizando 192 indivíduos por área. Para cada indivíduo marcado, foram medidos a distância até o ponto central do quadrante, por meio de trena, e o CAP (circunferência à altura do peito medida a 1,30 m do solo), com fita métrica, além de ser estimada a altura, por meio das varas do podão, e coletado o material botânico.
No levantamento florístico, foram incluídas todas as espécies amostradas nos pontos quadrantes, além de outras espécies localizadas em áreas adjacentes aos transectos por meio de excursões pela área, mantendo-se o mesmo critério de inclusão (DAP $\geq 3 \mathrm{~cm}$ ). O material botânico coletado foi herborizado e depositado no Herbário Montes Claros (HMC), da Universidade Estadual de Montes Claros (UNIMONTES). A identificação do material botânico coletado foi feita por meio de consultas a especialistas, uso de literatura especializada e comparações com as exsicatas do HMC. Para a classificação das espécies em famílias, foi utilizado o sistema do Angiosperm Phylogeny Group II (ANGIOSPERM PHYLOGENY GROUP-APG, 2003).

\subsection{Análise de dados}

Para descrição da estrutura da comunidade arbórea, foram calculados os seguintes parâmetros fitossociológicos (MUELLER-DOMBOIS \& ELLEMBERG, 1974; MARTINS, 1993):

Frequência absoluta (\%)

$F A(i)=\frac{j(i)}{k} \times 100$

Frequência relativa $(\%)$

$F R=\frac{F A(i)}{\sum_{i=1}^{n} F A} \times 100$ 
onde: $J(i)$ é o número de pontos que a espécie i foi observada e $K$ o número total de pontos na amostra.

Distância média (m)

$$
d_{\text {média }}=\frac{\sum d}{N}
$$

Área média $\left(\mathrm{m}^{2}\right)$

$$
A_{\text {média }}=\left(d_{\text {média }}\right)^{2}
$$

onde: $d$ é a distância medida entre o indivíduo registrado e o ponto (distância ponto-planta) e $N$ o número total de indivíduos registrados.

Densidade total amostrada (indiv.ha ${ }^{-1}$ )

$$
D T A=\frac{10.000}{A_{\text {média }}}
$$

Densidade absoluta (ind.ha ${ }^{-1}$ )

$$
D A=\frac{n(i)}{N} \times D T A
$$

Densidade relativa $(\%)$

$$
D R=\frac{D A(i)}{\sum_{i=1}^{n} D A} \times 100
$$

onde: $n(i)$ é o número de indivíduos amostrados para a espécie i e $N$ a abundância total da amostragem.

Dominância absoluta ( $\left.\mathrm{m}^{2} / \mathrm{ha}\right)$

$$
D o A=A B_{\text {média }}(i) \times D A(i)
$$

Dominância relativa (\%)

$$
D o R=\frac{D o A(i)}{\sum_{i=1}^{n} D o A} \times 100
$$

onde: $A B_{\text {média }}$ é a área basal média da espécie $\mathrm{i}$.

Valor de importância:

$$
V I=F R+D R+D o R
$$

Para estimar a diversidade das áreas estudadas, foram calculados ainda os índices de diversidade de Shannon $\left(H^{\prime}\right)$ e de equabilidade de Pielou $\left(J^{\prime}\right)$ (BEGON et al., 1996):

Cerne, Lavras, v. 16, n. 3, p. 267-281, jul./set. 2010
$H=-\sum_{i=1}^{s} P i \ln P i$

$j=\frac{H}{\ln S}$

onde: $P i$ é a proporção de indivíduos da espécie i em relação ao total e $S$ o total de número de espécies.

Os índices de diversidade de Shannon dos setores foram comparados pelo teste $t$ de Hutcheson (ZAR, 1996). Além disso, a riqueza de espécies entre os ambientes também foi comparada pelo estimador Jacknife 1 (BURNHAM \& OVERTON, 1979), calculado no programa EstimateS 8.2.0 (COLWELL, 2006).

A similaridade florística entre os fragmentos foi realizada por meio do índice de similaridade de Sorensen (Ss) (MUELLER-DOMBOIS \& ELLENBERG, 1974):

$$
I S=\frac{2 c}{A+B} \times 100
$$

onde: $A$ é número de espécies total da comunidade $\mathrm{A}, B$ o número de espécies total da comunidade $\mathrm{B}$ e $C$ é o número de espécies em comum.

Além disso, para se obter um melhor entendimento da estrutura da vegetação das áreas, foram elaborados histogramas de frequência de classes de diâmetro e de altura para todos os indivíduos amostrados, conforme proposto por Fidelis \& Godoy (2003).

\section{RESULTADOS E DISCUSSÃO}

No levantamento florístico, foram amostradas um total de 73 espécies, sendo que a área 1 contribuiu com 48 espécies, distribuídas em 41 gêneros e 24 famílias, e a área 2 com 54 espécies distribuídas em 47 gêneros e 28 famílias (desconsiderando-se as duas espécies não identificadas morfoespécies) (Tabela 1). A área 1 apresentou 19 espécies exclusivas e na área 2 foram encontradas 25. A maior riqueza encontrada na área 2 pode estar relacionada à sua localização em área de transição entre Cerrado Sentido Restrito e campo rupestre, característica confirmada pela presença da família Asteraceae com os gêneros Lychnophora e Vernonia. Além disso, o terreno íngreme dessa área dificulta seu desmatamento e sua conversão em pastagem. Assim, o fogo parece ser o principal agente perturbador desse ambiente, não ocorrendo com muita frequência. 
Florística e estrutura da comunidade arbórea ...

Tabela 1 - Famílias e espécies da flora arbórea amostradas em duas áreas de Cerrado Sentido Restrito em Grão Mogol, MG.

Table 1 - Families and species of arborous flora sampled in two sensu stricto cerrado areas vegetation from Grão Mogol, MG.

\begin{tabular}{|c|c|c|}
\hline \multirow{2}{*}{ Família/espécie } & \multicolumn{2}{|c|}{ Área } \\
\hline & 1 & 2 \\
\hline \multicolumn{3}{|l|}{ Anacardiaceae } \\
\hline Anacardium occidentale $\mathrm{L}$. & - & $\mathrm{X}$ \\
\hline Astronium fraxinifolium Schott ex Spreng. & - & $\mathrm{X}$ \\
\hline Lithraea molleoides (Vell.) Engl. & $\mathrm{X}$ & $\mathrm{X}$ \\
\hline \multicolumn{3}{|l|}{ Annonaceae } \\
\hline Annona coriacea Mart. & $\mathrm{X}$ & - \\
\hline \multicolumn{3}{|l|}{ Apocynaceae } \\
\hline Aspidosperma tomentosum Mart. & - & $\mathrm{X}$ \\
\hline Hancornia speciosa Gomes & - & $\mathrm{X}$ \\
\hline Himatanthus obovatus (Müll.Arg.) Woodson & - & $\mathrm{X}$ \\
\hline \multicolumn{3}{|l|}{ Araliaceae } \\
\hline Schefflera macrocarpa (Cham. \& Schltdl.) Frodin & - & $\mathrm{X}$ \\
\hline \multicolumn{3}{|l|}{ Asteraceae } \\
\hline Eremanthus erythropappus (DC.) Mc Leisch & - & $\mathrm{X}$ \\
\hline Lychnophora sp. & - & $\mathrm{X}$ \\
\hline Vernonia sp. & - & $\mathrm{X}$ \\
\hline \multicolumn{3}{|l|}{ Bignoniaceae } \\
\hline Tabebuia aurea (Manso) Benth. \& Hook.f. ex S.Moore & $\mathrm{X}$ & - \\
\hline \multicolumn{3}{|l|}{ Caryocaraceae } \\
\hline Caryocar brasiliense Cambess. & $\mathrm{X}$ & $\mathrm{X}$ \\
\hline \multicolumn{3}{|l|}{ Celastraceae } \\
\hline Plenckia populnea Reissek & $\mathrm{X}$ & $\mathrm{X}$ \\
\hline \multicolumn{3}{|l|}{ Clusiaceae } \\
\hline Kielmeyera coriacea Mart. \& Zucc. & - & $\mathrm{X}$ \\
\hline \multicolumn{3}{|l|}{ Combretaceae } \\
\hline Terminalia argentea (Cambess.) Mart. & $\mathrm{x}$ & $\mathrm{X}$ \\
\hline Terminalia fagifolia Mart. & - & $\mathrm{X}$ \\
\hline \multicolumn{3}{|l|}{ Dilleniaceae } \\
\hline Curatella americana $\mathrm{L}$. & $\mathrm{x}$ & - \\
\hline \multicolumn{3}{|l|}{ Erythroxylaceae } \\
\hline Erythroxylum tortuosum Mart. & $\mathrm{X}$ & - \\
\hline \multicolumn{3}{|l|}{ Euphorbiaceae } \\
\hline Maprounea guianensis Aubl. & $\mathrm{x}$ & - \\
\hline Pera glabrata (Schott) Poepp. ex Baill. & - & $\mathrm{X}$ \\
\hline
\end{tabular}

To be continued..

Cerne, Lavras, v. 16, n. 3, p. 267-281, jul./set. 2010 
Tabela 1 - Continua...

Table 1 - Continued...

Família/espécie

Fabaceae

Fabaceae sp. 1

Fabaceae sp. 2

Fabaceae Caesalpinioideae

Bauhinia pulchella Benth.

Copaifera langsdorffii Desf.

Hymenaea stigonocarpa Mart. ex Hayne

Tachigali aurea Tul.

Fabaceae Faboideae

Acosmium dasycarpum (Vogel) Yakovlev

Bowdichia virgilioides Kunth

Machaerium opacum Vogel

Swartzia sp.

Fabaceae Mimosoideae

Mimosa pteridifolia Benth.

Plathymenia reticulata Benth.

Stryphnodendron adstringens (Mart.) Cov.

Icacinaceae

Emmotum nitens (Benth.) Miers

Lamiaceae

Hyptidendron canum (Pohl) Harley

Loganiaceae

Strychnos pseudoquina A.St.-Hil.

Lythraceae

Lafoensia pacari A.St.-Hil.

Malphigiaceae

Byrsonima coccolobifolia Kunth

Byrsonima crassifolia (L.) Kunth

Heteropterys byrsonimifolia A.Juss.

Mascagnia sp.

Malpighiaceae sp.1

Malvaceae

Eriotheca pubescens (Mart. \& Zucc.) Schott \& Endl.

\begin{tabular}{lll}
\multicolumn{3}{c}{ Área } \\
\hline 1 & & 2
\end{tabular}

$\mathrm{X}$

$\mathrm{X}$

X $\quad$ X

$X \quad X$

X $\mathrm{X}$

$\mathrm{X}$

$\mathrm{X}$

$\mathrm{X}$

$\mathrm{X}$

$\mathrm{X}$

$\begin{array}{ll}- & X \\ - & X\end{array}$

X

$\mathrm{X}$

$\begin{array}{ll}- & X\end{array}$

$\mathrm{X}$

- $\quad$ X

X

$\mathrm{X}$

X $\quad \mathrm{X}$

X -

X -

$\mathrm{X}$
X

$\mathrm{X}$

$\mathrm{X}$

X

$\mathrm{X}$

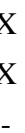

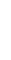

$\mathrm{X}$

$-$

$X$

$\mathrm{X}$

X

X

$\mathrm{X}$

$-$

$-$

X

Continua...

To be continued...

Cerne, Lavras, v. 16, n. 3, p. 267-281, jul./set. 2010 
Tabela 1 - Continua..

Table 1 - Continued...

Família/espécie $\quad$\begin{tabular}{l} 
Área \\
\cline { 2 - 3 }
\end{tabular}

Melastomataceae

Miconia sp.

X

2

Myrsinaceae

Myrsine guianensis (Aubl.) Kuntze

$\mathrm{X}$

$\mathrm{X}$

Myrtaceae

Eugenia dysenterica DC.

Myrcia tomentosa (Aubl.) DC.

Myrcia sp.

Psidium firmum O.Berg.

ctaginaceae

Guapira noxia (Netto) Lundell

Guapira sp.

Neea theifera Oerst.

Ochnaceae

Ouratea castaneifolia (DC.) Engl.

$X$

$\mathrm{X}$

X $\quad$ X

X -

$\mathrm{X} \quad \mathrm{X}$

$\mathrm{X}$

$\mathrm{X}$

$\mathrm{X}$

$\mathrm{X}$

$-\quad X$

- $\quad \mathrm{X}$

Proteaceae

Roupala montana Aubl.

$\begin{array}{ll}- & X\end{array}$

Rubiaceae

Alibertia sp.

X $\quad X$

$\mathrm{X} \quad \mathrm{X}$

Rutaceae

Zanthoxylum riedelianum Engl.

X

X

Salicaceae

Casearia sylvestris $\mathrm{Sw}$.

- X

Sapindaceae

Cupania vernalis Cambess.

Magonia pubescens A.St.-Hil.

Sapotaceae

Chrysophyllum marginatum (Hook. \& Arn.) Radlk.

Pouteria ramiflora (Mart.) Radlk.

X

$\mathrm{X}$

Solanaceae

Solanum lycocarpum A.St.-Hil.

$\mathrm{X}$

Continua...

To be continued...

Cerne, Lavras, v. 16, n. 3, p. 267-281, jul./set. 2010 
Tabela 1 - Continua...

Table 1 - Continued...

\begin{tabular}{lcc}
\multicolumn{1}{c}{ Família/espécie } & & Área \\
\cline { 2 - 3 } Vochysiaceae & 1 & 2 \\
Callisthene major Mart. & & \\
Qualea grandiflora Mart. & - & $\mathrm{X}$ \\
Qualea multiflora Mart. & $\mathrm{X}$ & $\mathrm{X}$ \\
Qualea parviflora Mart. & $\mathrm{X}$ & $\mathrm{X}$ \\
Salvertia convallariodora A.St.-Hil. & $\mathrm{X}$ & $\mathrm{X}$ \\
Vochysia rufa Mart. & - & $\mathrm{X}$ \\
Vochysia thyrsoidea Pohl & $\mathrm{X}$ & - \\
Não identificadas & $\mathrm{X}$ & $\mathrm{X}$ \\
sp. 1 & & $\mathrm{X}$ \\
sp. 2 & - & - \\
\hline
\end{tabular}

$\mathrm{Na}$ área 1, a família que apresentou maior número de espécies foi Fabaceae (11 espécies), seguida de Vochysiaceae (5), Malpighiaceae (5) e Myrtaceae (4), representando um total de $52,1 \%$ das espécies amostradas. $\mathrm{Na}$ área 2, as famílias mais representativas também foram Fabaceae (9 espécies) e Vochysiaceae (6), sendo, posteriormente, acrescidas de Nyctaginaceae, Anacardiaceae, Apocynaceae e Asteraceae, com três espécies cada que, juntas, perfizeram um total de 50,0\% das espécies inventariadas. As famílias Fabaceae e Vochysiaceae estão entre as principais famílias encontradas no Cerrado Sentido Restrito em Minas Gerais (NERI et al., 2007a), corroborando com a alta representatividade dessas famílias nas duas áreas. Espécies da família Fabaceae se destacam em áreas de Cerrado, em razão da sua capacidade de nodulação, conferindo adaptabilidade em regiões com baixo teor de nitrogênio, onde o solo, na maioria das vezes, é pobre em nutrientes (CORDEIRO, 2000). Segundo Haridassan \& Araújo (1988), muitas espécies de Vochysiaceae são acumuladoras de alumínio, proporcionando uma vantagem para crescer com sucesso nos solos do Cerrado (FELFILI \& SILVA-JÚNIOR, 1993).

A densidade total estimada para a área 1 foi de 1275,51 ind/ha (Tabela 2), caracterizando esse fragmento como Cerrado Sentido Restrito. De acordo com Felfili et al. (1994), as amplitudes de densidade dessa fitofisionomia estão entre 664-1396 ind/ha. A área 2 apresentou densidade total de 1580,58 ind/ha (Tabela 3). Apesar de apresentar um valor entre as amplitudes de 960-2082 ind/ha, o que pode caracterizar as duas áreas estudadas como de cerradão (FELFILI et al., 1994), o fragmento 2, especificamente, foi considerado como Cerrado Sentido Restrito Denso por não apresentar características fisionômicas florestais (NERI et al., 2007a). Estudos recentes revelam que a vegetação do Cerrado apresenta heterogeneidade entre as áreas. Essa heterogeneidade geralmente é atribuída à natureza geológica e geomorfológica, as quais geram diferenças fisionômicas, florísticas e estruturais (BORGES \& SHEPHERD, 2005).

Espécies que apresentam altos valores de importância (IVI) possuem maior sucesso na exploração dos recursos oferecidos pelo ambiente (ANDRADE et al., 2002). Dessa forma, na área 1 , as espécies com maiores valores de importância (IVI) foram Qualea parviflora $(29,2)$, Eriotheca pubescens $(23,8)$, Vochysia thyrsoidea $(17,0)$ Eugenia dysenterica $(16,2)$ e Tabebuia aurea $(16,1)$ (Tabela 2). Estes IVIs estão associados aos altos valores de frequência e densidade relativas apresentados por $Q$. parviflora $(12,7$ e 14,6; respectivamente), E. pubescens (10,9 e 9,4), E. dysenterica $(6,1$ e 7,8$)$ e $T$. aurea $(6,1$ e 6,3), e alto valor de dominância relativa apresentado por $V$. thyrsoidea $(15,9)$. Essas cinco espécies correspondem a $34,1 \%$ do IVI e 38,5\% dos indivíduos amostrados. Desse grupo, $Q$. parviflora e T. aurea são espécies recorrentes nos levantamentos realizados na área nuclear do Cerrado (RIBEIRO \& WALTER, 1998). Além disso, Q. parviflora está entre as espécies de maior IVI em outros estudos (BALDUINO et al., 2005; SILVA et al., 2002).

Cerne, Lavras, v. 16, n. 3, p. 267-281, jul./set. 2010 
Tabela 2 - Parâmetros fitossociológicos das espécies arbóreas amostradas na área 1 de Cerrado Sentido Restrito em Grão Mogol, MG. $\mathrm{N}=$ número de indivíduos; $\mathrm{A}=$ absoluta; $\mathrm{R}=$ relativa .

Table 2 - Woody species phytosociological parameters sampled in area 1 of sensu stricto cerrado vegetation in Grão Mogol, MG. $N=$ number of individuals; $A=$ absolute; $R=$ relative.

\begin{tabular}{|c|c|c|c|c|c|c|c|c|}
\hline \multirow{2}{*}{ Espécie } & \multirow{2}{*}{$\mathrm{N}$} & \multicolumn{2}{|c|}{ Densidade } & \multicolumn{2}{|c|}{ Dominância } & \multicolumn{2}{|c|}{ Frequência } & \multirow{2}{*}{ IVI } \\
\hline & & $\mathrm{A}(\mathrm{n} / \mathrm{ha})$ & $\mathrm{R}(\%)$ & $\mathrm{A}\left(\mathrm{m}^{2} / \mathrm{ha}\right)$ & $\mathrm{R}(\%)$ & $\mathrm{A}(\%)$ & $\mathrm{R}(\%)$ & \\
\hline Qualea parviflora & 28 & 186,01 & 14,58 & 0,02 & 1,90 & 0,44 & 12,72 & 29,20 \\
\hline Eriotheca pubescens & 18 & 119,58 & 9,38 & 0,04 & 3,53 & 0,38 & 10,90 & 23,81 \\
\hline Vochysia thyrsoidea & 1 & 6,64 & 0,52 & 0,18 & 15,86 & 0,02 & 0,61 & 16,99 \\
\hline Eugenia dysenterica & 15 & 99,65 & 7,81 & 0,03 & 2,35 & 0,21 & 6,06 & 16,22 \\
\hline Tabebuia aurea & 12 & 79,72 & 6,25 & 0,04 & 3,74 & 0,21 & 6,06 & 16,05 \\
\hline Machaerium opacum & 11 & 73,08 & 5,73 & 0,02 & 2,14 & 0,21 & 6,06 & 13,93 \\
\hline Pouteria ramiflora & 3 & 19,93 & 1,56 & 0,11 & 9,43 & 0,06 & 1,82 & 12,81 \\
\hline Magonia pubescens & 11 & 73,08 & 5,73 & 0,01 & 0,98 & 0,19 & 5,45 & 12,16 \\
\hline Caryocar brasiliense & 4 & 26,57 & 2,08 & 0,09 & 7,54 & 0,08 & 2,42 & 12,05 \\
\hline Terminalia argentea & 9 & 59,79 & 4,69 & 0,04 & 3,37 & 0,13 & 3,63 & 11,69 \\
\hline Heteropterys byrsonimifolia & 10 & 66,43 & 5,21 & 0,01 & 0,94 & 0,19 & 5,45 & 11,60 \\
\hline Lafoensia pacari & 9 & 59,79 & 4,69 & 0,04 & 3,16 & 0,13 & 3,63 & 11,48 \\
\hline Hymenaea stigonocarpa & 7 & 46,50 & 3,65 & 0,03 & 2,59 & 0,13 & 3,63 & 9,87 \\
\hline Copaifera langsdorffii & 3 & 19,93 & 1,56 & 0,07 & 6,02 & 0,06 & 1,82 & 9,40 \\
\hline Acosmium dasycarpum & 6 & 39,86 & 3,13 & 0,02 & 2,02 & 0,13 & 3,63 & 8,78 \\
\hline Qualea grandiflora & 6 & 39,86 & 3,13 & 0,01 & 1,24 & 0,10 & 3,03 & 7,39 \\
\hline Guapira noxia & 3 & 19,93 & 1,56 & 0,03 & 2,71 & 0,06 & 1,82 & 6,09 \\
\hline Qualea multiflora & 4 & 26,57 & 2,08 & 0,01 & 0,91 & 0,08 & 2,42 & 5,42 \\
\hline Hyptidendron canum & 3 & 19,93 & 1,56 & 0,03 & 2,73 & 0,04 & 1,21 & 5,51 \\
\hline Byrsonima crassifolia & 1 & 6,64 & 0,52 & 0,05 & 4,12 & 0,02 & 0,61 & 5,24 \\
\hline Chamaecrista sp. & 4 & 26,57 & 2,08 & 0,01 & 0,72 & 0,08 & 2,42 & 5,23 \\
\hline Tocoyena formosa & 3 & 19,93 & 1,56 & 0,02 & 1,71 & 0,06 & 1,82 & 5,09 \\
\hline Bowdichia virgilioides & 1 & 6,64 & 0,52 & 0,04 & 3,57 & 0,02 & 0,61 & 4,70 \\
\hline Ouratea castaneifolia & 2 & 13,29 & 1,04 & 0,02 & 1,57 & 0,04 & 1,21 & 3,82 \\
\hline Stryphnodendron adstringens & 2 & 13,29 & 1,04 & 0,02 & 1,37 & 0,04 & 1,21 & 3,63 \\
\hline Tachigali aurea & 1 & 6,64 & 0,52 & 0,03 & 2,39 & 0,02 & 0,61 & 3,52 \\
\hline Byrsonima coccolobifolia & 2 & 13,29 & 1,04 & 0,01 & 1,23 & 0,04 & 1,21 & 3,49 \\
\hline Vochysia rufa & 2 & 13,29 & 1,04 & 0,01 & 0,89 & 0,04 & 1,21 & 3,15 \\
\hline Myrsine guianensis & 2 & 13,29 & 1,04 & 0,01 & 0,68 & 0,04 & 1,21 & 2,94 \\
\hline Cupania vernalis & 1 & 6,64 & 0,52 & 0,02 & 1,73 & 0,02 & 0,61 & 2,86 \\
\hline Myrcia tomentosa & 2 & 13,29 & 1,04 & 0,01 & 0,60 & 0,04 & 1,21 & 2,86 \\
\hline Fabaceae sp. 1 & 1 & 6,64 & 0,52 & 0,02 & 1,48 & 0,02 & 0,61 & 2,60 \\
\hline Myrcia sp. & 1 & 6,64 & 0,52 & 0,02 & 1,48 & 0,02 & 0,61 & 2,60 \\
\hline Plenckia populnea & 1 & 6,64 & 0,52 & 0,01 & 1,21 & 0,02 & 0,61 & 2,33 \\
\hline Mascagnia sp. & 1 & 6,64 & 0,52 & 0,01 & 1,03 & 0,02 & 0,61 & 2,15 \\
\hline Malpighiaceae sp. 1 & 1 & 6,64 & 0,52 & 0,01 & 0,60 & 0,02 & 0,61 & 1,73 \\
\hline Swartzia sp. & 1 & 6,64 & 0,52 & 0,01 & 0,46 & 0,02 & 0,61 & 1,58 \\
\hline Total & 192 & 1275,51 & 100,0 & 1,16 & 100,0 & 3,44 & 100,0 & 300,0 \\
\hline
\end{tabular}


Tabela 3 - Parâmetros fitossociológicos das espécies arbóreas amostradas na área 2 de Cerrado Sentido Restrito em Grão Mogol, MG. A = absoluta; $\mathrm{R}$ = relativa.

Table 3 - Woody species phytosociological parameters sampled in area 2 of sensu stricto cerrado vegetation in Grão Mogol, MG. $A=$ absolute $R=$ relative.

\begin{tabular}{|c|c|c|c|c|c|c|c|c|}
\hline \multirow{2}{*}{ Espécie } & \multirow{2}{*}{$\mathrm{N}$} & \multicolumn{2}{|c|}{ Densidade } & \multicolumn{2}{|c|}{ Dominância } & \multicolumn{2}{|c|}{ Frequência } & \multirow{2}{*}{ IVI } \\
\hline & & $\mathrm{A}(\mathrm{n} / \mathrm{ha})$ & $\mathrm{R}(\%)$ & $\mathrm{A}\left(\mathrm{m}^{2} / \mathrm{ha}\right)$ & $\mathrm{R}(\%)$ & $\mathrm{A}(\%)$ & $\mathrm{R}(\%)$ & \\
\hline Qualea parviflora & 35 & 288,14 & 18,23 & 0,03 & 2,12 & 0,54 & 15,13 & 35,48 \\
\hline Eriotheca pubescens & 16 & 131,72 & 8,33 & 0,07 & 4,15 & 0,27 & 7,57 & 20,05 \\
\hline Guapira sp. & 14 & 115,26 & 7,29 & 0,02 & 1,08 & 0,27 & 7,57 & 15,93 \\
\hline Roupala montana & 11 & 90,56 & 5,73 & 0,03 & 1,77 & 0,21 & 5,82 & 13,32 \\
\hline Terminalia fagifolia & 10 & 82,33 & 5,21 & 0,03 & 2,11 & 0,19 & 5,24 & 12,55 \\
\hline Vochysia thyrsoidea & 3 & 24,70 & 1,56 & 0,13 & 7,97 & 0,06 & 1,75 & 11,28 \\
\hline Acosmium dasycarpum & 8 & 65,86 & 4,17 & 0,02 & 1,22 & 0,17 & 4,66 & 10,04 \\
\hline Plenckia populnea & 7 & 57,63 & 3,65 & 0,03 & 1,88 & 0,15 & 4,07 & 9,60 \\
\hline Qualea grandiflora & 7 & 57,63 & 3,65 & 0,05 & 2,78 & 0,10 & 2,91 & 9,34 \\
\hline Aspidosperma tomentosum & 2 & 16,47 & 1,04 & 0,12 & 7,10 & 0,04 & 1,16 & 9,31 \\
\hline Eremanthus erythropappus & 6 & 49,40 & 3,13 & 0,04 & 2,27 & 0,13 & 3,49 & 8,88 \\
\hline Caryocar brasiliense & 2 & 16,47 & 1,04 & 0,11 & 6,64 & 0,04 & 1,16 & 8,84 \\
\hline Guapira noxia & 7 & 57,63 & 3,65 & 0,02 & 1,24 & 0,13 & 3,49 & 8,38 \\
\hline Astronium fraxinifolium & 1 & 8,23 & 0,52 & 0,11 & 6,77 & 0,02 & 0,58 & 7,87 \\
\hline Myrsine guianensis & 3 & 24,70 & 1,56 & 0,06 & 3,82 & 0,06 & 1,75 & 7,13 \\
\hline Lafoensia pacari & 5 & 41,16 & 2,60 & 0,03 & 1,58 & 0,10 & 2,91 & 7,09 \\
\hline Lychnophora sp. & 5 & 41,16 & 2,60 & 0,02 & 1,46 & 0,10 & 2,91 & 6,97 \\
\hline Myrcia sp. & 5 & 41,16 & 2,60 & 0,03 & 1,55 & 0,08 & 2,33 & 6,49 \\
\hline Miconia sp. & 2 & 16,47 & 1,04 & 0,06 & 3,51 & 0,04 & 1,16 & 5,71 \\
\hline Hancornia speciosa & 4 & 32,93 & 2,08 & 0,02 & 1,23 & 0,08 & 2,33 & 5,64 \\
\hline Não identificada sp. 2 & 5 & 41,16 & 2,60 & 0,01 & 0,68 & 0,08 & 2,33 & 5,61 \\
\hline Anacardium occidentale & 1 & 8,23 & 0,52 & 0,07 & 4,38 & 0,02 & 0,58 & 5,49 \\
\hline Dalbergia miscolobium & 3 & 24,70 & 1,56 & 0,03 & 1,65 & 0,06 & 1,75 & 4,96 \\
\hline Copaifera langsdorffii & 3 & 24,70 & 1,56 & 0,02 & 1,34 & 0,06 & 1,75 & 4,65 \\
\hline Callisthene major & 1 & 8,23 & 0,52 & 0,06 & 3,48 & 0,02 & 0,58 & 4,58 \\
\hline Machaerium opacum & 1 & 8,23 & 0,52 & 0,05 & 3,35 & 0,02 & 0,58 & 4,45 \\
\hline Kielmeyera coriacea & 2 & 16,47 & 1,04 & 0,02 & 1,53 & 0,04 & 1,16 & 3,74 \\
\hline Zanthoxylum riedelianum & 1 & 8,23 & 0,52 & 0,04 & 2,52 & 0,02 & 0,58 & 3,62 \\
\hline Tocoyena formosa & 2 & 16,47 & 1,04 & 0,02 & 1,05 & 0,04 & 1,16 & 3,26 \\
\hline Eugenia dysenterica & 1 & 8,23 & 0,52 & 0,03 & 2,13 & 0,02 & 0,58 & 3,23 \\
\hline Terminalia argentea & 1 & 8,23 & 0,52 & 0,03 & 2,13 & 0,02 & 0,58 & 3,23 \\
\hline Não identificada sp.1 & 2 & 16,47 & 1,04 & 0,01 & 0,68 & 0,04 & 1,16 & 2,89 \\
\hline Himatanthus obovatus & 1 & 8,23 & 0,52 & 0,03 & 1,78 & 0,02 & 0,58 & 2,88 \\
\hline
\end{tabular}


Tabela 3 - Continua...

Table 3 - Continued...

\begin{tabular}{|c|c|c|c|c|c|c|c|c|}
\hline \multirow{2}{*}{ Espécie } & \multirow{2}{*}{$\mathrm{N}$} & \multicolumn{2}{|c|}{ Densidade } & \multicolumn{2}{|c|}{ Dominância } & \multicolumn{2}{|c|}{ Frequência } & \multirow{2}{*}{ IVI } \\
\hline & & $\mathrm{A}(\mathrm{n} / \mathrm{ha})$ & $\mathrm{R}(\%)$ & $\mathrm{A}\left(\mathrm{m}^{2} / \mathrm{ha}\right)$ & $\mathrm{R}(\%)$ & $\mathrm{A}(\%)$ & $\mathrm{R}(\%)$ & \\
\hline Neea theifera & 1 & 8,23 & 0,52 & 0,03 & 1,78 & 0,02 & 0,58 & 2,88 \\
\hline Ouratea castaneifolia & 1 & 8,23 & 0,52 & 0,02 & 1,11 & 0,02 & 0,58 & 2,22 \\
\hline Schefflera macrocarpa & 1 & 8,23 & 0,52 & 0,02 & 1,03 & 0,02 & 0,58 & 2,13 \\
\hline Byrsonima crassifolia & 1 & 8,23 & 0,52 & 0,02 & 0,97 & 0,02 & 0,58 & 2,08 \\
\hline Fabaceae sp. 2 & 1 & 8,23 & 0,52 & 0,01 & 0,79 & 0,02 & 0,58 & 1,89 \\
\hline Strychnos pseudoquina & 1 & 8,23 & 0,52 & 0,01 & 0,69 & 0,02 & 0,58 & 1,79 \\
\hline Vernonia sp. & 1 & 8,23 & 0,52 & 0,01 & 0,68 & 0,02 & 0,58 & 1,78 \\
\hline Stryphnodendron adstringens & 1 & 8,23 & 0,52 & 0,01 & 0,66 & 0,02 & 0,58 & 1,76 \\
\hline Hyptidendron canum & 1 & 8,23 & 0,52 & 0,01 & 0,58 & 0,02 & 0,58 & 1,68 \\
\hline Plathymenia reticulata & 1 & 8,23 & 0,52 & 0,01 & 0,58 & 0,02 & 0,58 & 1,68 \\
\hline Pouteria ramiflora & 1 & 8,23 & 0,52 & 0,01 & 0,49 & 0,02 & 0,58 & 1,59 \\
\hline Hymenaea stigonocarpa & 1 & 8,23 & 0,52 & 0,01 & 0,49 & 0,02 & 0,58 & 1,59 \\
\hline Chamaecrista sp. & 1 & 8,23 & 0,52 & 0,01 & 0,41 & 0,02 & 0,58 & 1,51 \\
\hline Pera glabrata & 1 & 8,23 & 0,52 & 0,01 & 0,40 & 0,02 & 0,58 & 1,51 \\
\hline Qualea multiflora & 1 & 8,23 & 0,52 & 0,01 & 0,39 & 0,02 & 0,58 & 1,49 \\
\hline Total & 192 & 1580,67 & 100,0 & 1,6274 & 100,0 & 3,58 & 100,0 & 300,0 \\
\hline
\end{tabular}

As espécies com maiores valores de IVI, da área 2, foram $Q$. parviflora $(35,5)$, E. pubescens $(20,1)$, Guapira sp. $(15,9)$, Roupala montana $(13,3)$ e Terminalia fagifolia $(12,6)$ (Tabela 3), representando $32,4 \%$ do IVI total e $37,0 \%$ dos indivíduos amostrados. Do mesmo modo, os altos valores de frequência relativa apresentados por $Q$. parviflora $(15,1)$, E. pubescens $(7,6)$, Guapira sp. $(7,6), R$. montana $(5,3)$ e $T$. fagifolia $(5,3)$ e também os elevados valores de densidade relativa apresentados pelas mesmas espécies $(18,2 ; 8,3 ; 7,3$; 5,7 e 5,2; respectivamente), determinaram a posição dessas espécies em relação ao IVI. Cabe ressaltar que $Q$. parviflora foi também a espécie mais abundante e frequente na área 2 , com 13,6\% da amostragem de indivíduos nesta área. Essa espécie distribui-se tanto em áreas de Cerrado Sentido Restrito, cerradão e até mesmo em campo cerrado (embora com densidade menores), conforme as observações de Campos et al. (2006).

Vochysia thyrsoidea foi a espécie que apresentou maior valor de dominância relativa (DoR), tanto na área 1 $(15,9 \%)$ quanto na área $2(7,9 \%)$, apesar de ter sido representada somente por um e três indivíduos, respectivamente. Algumas espécies, apesar de representadas por apenas um ou poucos indivíduos, destacam-se em relação aos valores de dominância em função dos elevados diâmetros inerentes (BORÉM \& OLIVEIRA-FILHO, 2002).

$\mathrm{O}$ índice de diversidade de Shannon foi semelhante entre as áreas $\left(H^{\prime}{ }_{1}=3,13 ; H^{\prime}{ }_{2}=3,27\right.$; área 1 e 2 respectivamente; $t=-1,270, P>0,05)$, assim como a equabilidade de Pielou $\left(J_{1}{ }_{1}=0,87 ; J_{2}=0,84\right.$, área 1 e 2 , respectivamente), reforçando a similaridade das áreas e enquadrando-as dentro da faixa de variação $(3,16$ a 3,73) de outros estudos no Cerrado (ASSUNÇÃO \& FELFILI, 2004; FELFILI \& SILVA-JUNIOR, 2001). Esses estudos corroboram os resultados do presente trabalho, evidenciando elevada diversidade de espécies para as áreas de estudo. $\mathrm{O}$ valor de equabilidade obtido indica que essas áreas apresentam considerável distribuição dos indivíduos por espécie, o que é confirmado pela ausência de dominância de um grupo de espécies em relação às demais.

Apesar da coleta do mesmo número de indivíduos nas duas áreas (192), o estimador de riqueza de espécies utilizado estimou aproximadamente 47 e 68 espécies, respectivamente, para as áreas 1 e 2 . Desconsiderando as coletas realizadas fora dos pontos quadrantes, os mesmos

Cerne, Lavras, v. 16, n. 3, p. 267-281, jul./set. 2010 
registraram 37 espécies para área 1 e 47 espécies para área 2. Assim, a riqueza observada foi menor do que a estimada, indicando que não foi atingida a suficiência amostral. As curvas de rarefação (Figura 2) não demostraram tendência a estabilização, principalmente na área 2. Entretanto, segundo Schilling \& Batista (2008), a determinação da suficiência amostral apresenta grande limitações, uma vez que, o aumento do número de unidades amostrais pode levar a mudança de ecounidades, causando a inclusão de novas espécies. Assim, apesar dos resultados encontrados, a validação ou não da amostragem não pode ser ponderada pelas análises realizadas.

A similaridade florística baseada no índice de Sorensen foi de 0,68 , valor considerável ao se avaliar duas áreas distintas. Esse valor está de acordo com o encontrado por Felfili et al. (1993) ao trabalhar em seis áreas de Cerrado Sentido Restrito cuja similaridade esteve entre 0,51 e 0,77. Entretanto, como são áreas muito próximas entre si $( \pm 1 \mathrm{~km})$, as diferenças encontradas, provavelmente, podem estar relacionadas às distintas pressões antrópicas que estas áreas têm sofrido e, consequentemente, têm levado à respostas desiguais na regeneração natural.

A distribuição diamétrica e de altura das áreas apresentaram a forma de J-invertido (Figura 3). Esse padrão de curva também foi observado em outros estudos (ANDRADE et al., 2002; SILVA-JÚNIOR, 2005). As áreas apresentam principalmente indivíduos jovens, com 74,5\% dos indivíduos da área 1 e 75,0\% da área 2 representados por diâmetros (DAP) inferiores a $10 \mathrm{~cm}$. Quanto à altura, 67,7\% dos indivíduos da área 1 e 77,6\% da área 2 apresentam altura abaixo de $4 \mathrm{~m}$. Esses resultados indicam que a vegetação das áreas estudadas é composta por indivíduos jovens. Esse fato pode ser observado em vários estudos, que demonstram que a concentração de indivíduos nas classes inferiores de altura e diâmetro denotam fase inicial de sucessão (FIDELIS \& GODOY, 2003; CARVALHO \& MARQUES-ALVES, 2008; SILVA-JÚNIOR \& SILVA, 1988). Entretanto, segundo Costa \& Araújo (2007), a ausência de informações detalhadas sobre o histórico de perturbações (inclusive do presente estudo) e a grande variação de critérios de inclusão de indivíduos nos diferentes trabalhos, compromete as inferências sobre a estrutura das comunidades e dos efeitos geradores (solo, microclima, ações antrópicas, etc.) dos padrões encontrados.

A altura máxima encontrada na área 1 de $9,5 \mathrm{~m}$ pertence a um indivíduo de V. thyrsoidea. Essa espécie também se destacou quanto sua altura em um estudo de Assunção \& Felfili (2004) em uma área de cerrado, no Distrito Federal. Na área 2, a altura máxima de $10 \mathrm{~m}$ pertenceu a um indivíduos de E. pubescens. O valor máximo de diâmetro obtido na área 1 foi $18,78 \mathrm{~cm}$, e na área 2,14,16 cm e também pertencia a dois indivíduos de V. thyrsoidea, corroborando os resultados de Assunção $\&$ Felfili (2004).

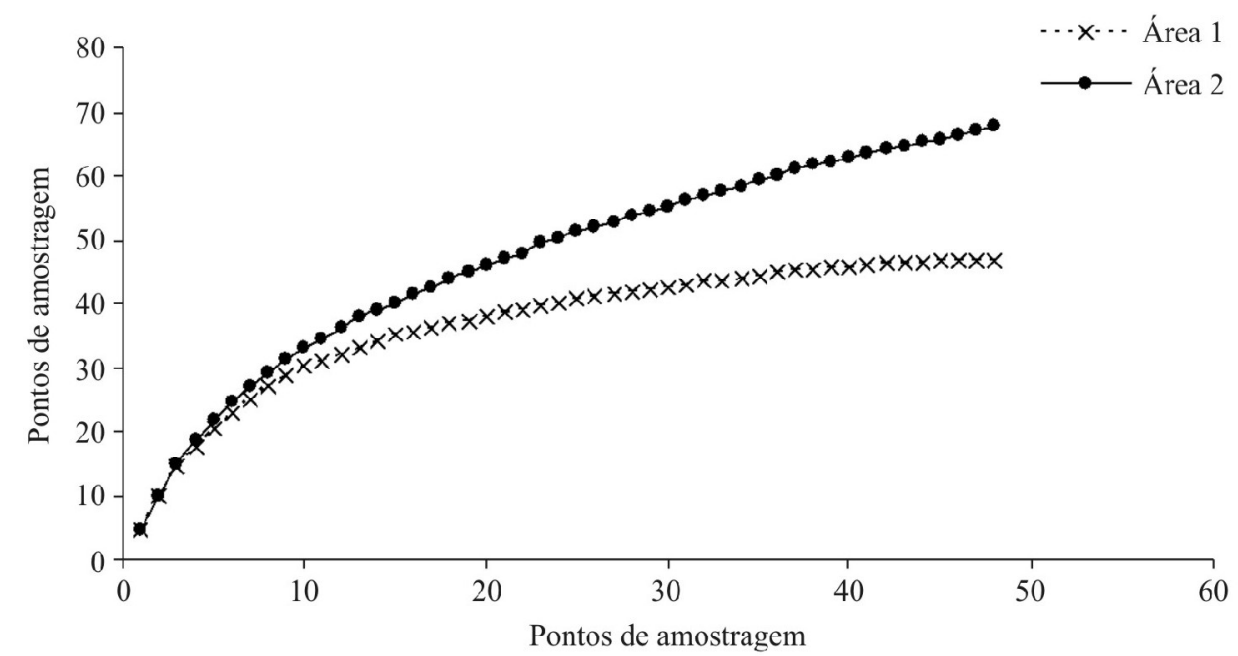

Figura 2 - Curvas de rarefação de estimador de riqueza (Jacknife 1) das áreas de Cerrado Sentido Restrito em Grão Mogol (MG), amostradas por meio de 48 pontos quadrantes.

Figure 2 - Rarefaction curve of richness estimators (Jacknife) of the Cerrado Stricto Sensu in Grão Mogol (MG), sampled by 48 quadrants points.

Cerne, Lavras, v. 16, n. 3, p. 267-281, jul./set. 2010 

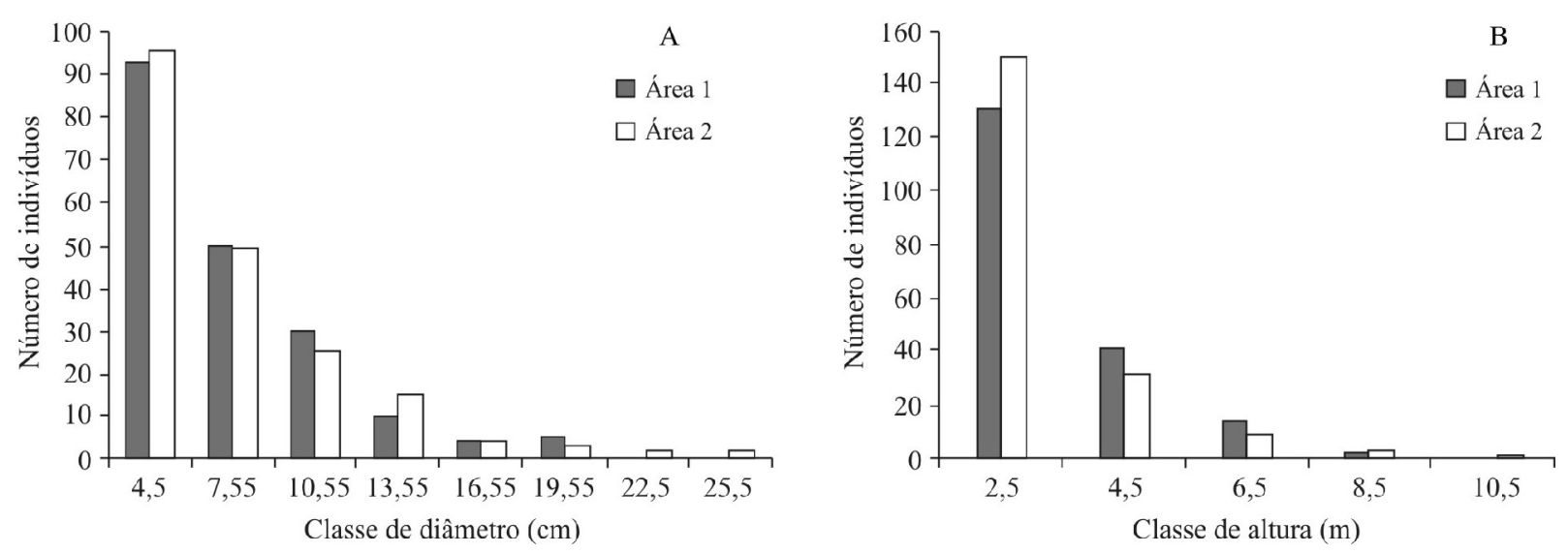

Figura 3 - Distribuição dos indivíduos amostrados em classes de diâmetro (A) e altura (B) em duas áreas de Cerrado Sentido Restrito no norte de Minas Gerais.

Figure 3 - Individuals distribution in diameter $(A)$ and height $(B)$ classes in two areas of cerrado stricto sensu in North of Minas Gerais.

A estrutura diamétrica revelou que as comunidades arbóreas estudadas são compostas principalmente por árvores pequenas. Felfili (1997) encontrou, na Mata do Gama (UNB, DF), 90\% das árvores com DAP menores de $45 \mathrm{~cm}$ e o diâmetro máximo de aproximadamente $100 \mathrm{~cm}$. O padrão da curva em J invertido, com a maioria das árvores localizadas na primeira classe de diâmetro $(3-6 \mathrm{~cm})$, indica um balanço positivo entre recrutamento e mortalidade $\mathrm{e}$ caracteriza a comunidade como autoregenerativa (SILVAJÚNIOR, 2005), caso não continue ocorrendo perturbações.

\section{CONCLUSÕES}

O total de espécies encontradas neste estudo (73) corrobora informações sobre o padrão de riqueza de espécies lenhosas do Cerrado Sentido Restrito. As Famílias Fabaceae e Vochysiaceae e as espécies $Q$. parviflora e $E$. pubescens foram as mais representativas em ambas as áreas, suportadas pelos altos valores de densidade e dominância fitossociológicas.

As áreas apresentam-se em processo de sucessão secundária, provavelmente desencadeados por eventos passados de perturbação, confirmados pela estrutura de tamanhos observada, com predomínio de indivíduos jovens. Apesar disto, a diversidade e equabilidade encontradas, somadas aos parâmetros fitossociológicos, indicam elevada diversidade florística local.

Apesar de elevada similaridade entre os ambientes estudados, a área 2 apresentou maiores índice de diversidade e equabilidade, e número de espécies e indivíduos, indicando um estágio sucessional mais avançado.

\section{AGRADECIMENTOS}

À FAPEMIG pela bolsa BICDT de Y. R. F. Nunes, de Doutorado de S. D'Angelo-Neto, de Mestrado de G. C. O. Menino, de Iniciação Científica de D. O. Brandão. À CAPES pelas bolsas de Mestrado de F. V. Costa e K. N. Oliveira. À UNIMONTES pelo apoio logístico. Ao Prof. César Vinícius Mendes Neves (Fundação Santo Agostinho) pela confecção do mapa de localização da área de estudo. Ao Luiz Dolabela Falcão pelo auxílio nas análises de dados. Aos revisores anônimos do artigo pelas críticas e sugestões, contribuindo para a versão final do mesmo. Este trabalho foi desenvolvido durante a disciplina Ecologia Especial do curso de Ciências Biológicas - Bacharelado da UNIMONTES.

\section{REFERÊNCIAS BIBLIOGRÁFICAS}

ANDRADE, L. A. Z.; FELFILI, J. M.; VIOLATTI, L. Fitossociologia de uma área de cerrado denso na RECOR-IBGE, Brasília-DF. Acta Botanica Brasilica, São Paulo, v. 16, n. 2, p. 225-240, 2002.

ANGIOSPERM PHYLOGENY GROUP. An update of the angiosperm phylogeny group classification for orders and families of flowering plants: APG II. Botanical Journal of the Linnean Society, v. 141, p. 399-436, 2003.

Cerne, Lavras, v. 16, n. 3, p. 267-281, jul./set. 2010 
ASSUNÇÃO, S. L.; FELFILI, J. M. Fitossociologia de um fragmento de cerrado sensu stricto na APA de Paranoá, DF, Brasil. Acta Botanica Brasilica, São Paulo, v. 18, n. 4, p. 903-910, 2004.

BALDUINO, A. P. C.; SOUZA, A. L.; MEIRA NETO, J. A. A.; SILVA, A. F.; SILVA JÚNIOR, M. C. Fitossociologia e análise comparativa da composição florística do cerrado da flora de Paraopeba-MG. Revista Árvore, Viçosa, v. 29, n. 1, p. 25-34, 2005.

BEGON, M.; HARPER, J. L.; TOWNSEND, C. R. Ecology: individuals, populations and communities. London: Blackwell Science, 1996. 1068 p.

BORÉM, R. A. T.; OLIVEIRA-FILHO, A. T. Fitossociologia do estrato arbóreo em uma topossequencia alterada de mata atlântica, no Município de Silva Jardim, RJ, Brasil. Revista Árvore, Viçosa, v. 26 , n. 6 , p. $727-742,2002$.

BORGES, H.; SHEPHERD, G. J. Flora e estrutura do estrato lenhoso numa comunidade de cerrado em Santo Antônio do Leverger, MT, Brasil. Revista Brasileira de Botânica, São Paulo, v. 28, p. 61-74, 2005.

BURNHAM, K. P.; OVERTON, W. S. Robust estimation of population size when capture probabilities vary among animals. Ecology, Durham, v. 60, n. 5, p. 927-936, 1979.

CAMPOS, E. P.; DUARTE, T. G.; NERI, A. V.; SILVA, A. F.; MEIRA-NETO, J. A. A.; VALENTE, G. E. Composição florística de um trecho de cerrado e cerradão sensu stricto e sua relação com o solo na Floresta Nacional (Flona) de Paraopeba, MG, Brasil. Revista Árvore, Viçosa, v. 30, n. 3, p. 471-479, 2006.

CARVALHO, A. R.; MARQUES-ALVES, S. Diversidade e índice sucessional de uma vegetação de cerrado sensu stricto na Universidade Estadual de Goiás-UEG, Campus de Anápolis. Revista Árvore, Viçosa, v. 32, n. 1, p. 81-90, 2008.

COLWELL, R. K. EstimateS: statistical estimation of species richness and shared species from samples. Version 8. Disponível em: 〈purl.oclc.org/estimates〉. Acesso em: 21 abr. 2009.

CONSERVAÇÃO INTERNACIONAL DO BRAZIL. Hotspots. 2009. rhttp://www.conservation.org.br/arquivos/Mapa $\%$ 'Hotspots\%202005.pdffi. Acesso em: 20 abr. 2009.

Cerne, Lavras, v. 16, n. 3, p. 267-281, jul./set. 2010
CORDEIRO, L. Fixação de nitrogênio em leguminosas ocorrentes no cerrado. In: KLEIN, A. L. (Org.). Eugene Warming e o Cerrado brasileiro: um século depois. São Paulo: Universidade Estadual de São Paulo, 2000. p. 131-145.

COSTA, I. R.; ARAÚJO, F. S. Organização comunitária de um encrave de cerrado sensu stricto no bioma Caatinga, chapada do Araripe, Barbalha, Ceará. Acta Botanica Brasilica, São Paulo, v. 21, n. 2, p. 281-291, 2007.

COTTAM, G.; CURTIS, J. T. The use of distance measures in phytosociological sampling. Ecology, New York, v. 37, n. 3, p. 451-460, 1956.

COUTINHO, L. M. O conceito de Cerrado. Revista Brasileira de Botânica, São Paulo, v. 1, p. 17-24, 1978.

DURIGAN, G. Métodos para análise de vegetação arbórea. In: CULLEN JUNIOR, L. (Ed.). Métodos de estudo em biologia da conservação e manejo da vida silvestre. Curitiba: UFPR, 2004. p. 455-480.

EITEN, G. Vegetação do Cerrado. In: NOVAES PINTO, M. (Org.). Cerrado. 2. ed. Brasília: UnB, 1993. p. 17-73.

FELFILI, J. M. Diameter and height distributions in a galery forest tree community and some of its main species in Central Brazil over a six year period, 1985-1991. Revista Brasileira de Botânica, São Paulo, v. 20, p. 155-162, 1997.

FELFILI, M. C.; FELFILI, J. M. Diversidade alfa e beta no cerrado sensu stricto da Chapada Pratinha, Brasil. Acta Botanica Brasilica, São Paulo, v. 15, n. 2, p. 243-254, 2001.

FELFILI, J. M.; HARIDASAN, M.; MENDONÇA, R. C. Projeto biogeografia do bioma cerrado: vegetação e solos. Caderno de Geografia, Rio de Janeiro, n. 12, p. 75-176, 1994.

FELFILI, J. M.; NASCIMENTO, A. R. T.; FAGG, C. W.; MEIRELLES, E. M. Floristic composition and community structure of a seasonally deciduous forest on limestone outcrops in Central Brazil. Revista Brasileira de Botânica, São Paulo, v. 30, p. 611-621, 2007.

FELFILI, J. M.; NOGUEIRA, P. E.; SILVA-JUNIOR, M. C.; MARIMON, B. S.; DELITTI, W. B. C. Composição florística e fitossociológica do Cerrado sentido restrito no município de Água Boa, MT. Acta Botanica Brasilica, São Paulo, v. 16, n. 1, p. 103-112, 2002. 
FELFILI, J. M.; SILVA-JÚNIOR, M. C. A comparative study of cerrado (sensu stricto) vegetation in Central Brazil. Journal of Tropical Ecology, Cambridge, v. 9, p. 277-289, 1993.

FELFILI, J. M.; SILVA-JUNIOR, M. C. Biogeografia do bioma Cerrado: estudo fitofisionômino na Chapada do Espigão Mestre do São Francisco. Brasília: UnB, 2001. 152 p.

FELFILI, J. M.; SILVA-JÚNIOR, M. C.; REZENDE, A. V.; MACHADO, B. W. T.; SILVA, P. E. N.; HAY, J. D. Análise comparativa de florística e fitossociologia da vegetação arbórea do cerrado sensu stricto na Chapada Pratinha, Brasil. Acta Botanica Brasilica, São Paulo, v. 6, n. 2, p. 27-46, 1993.

FELFILI, J. M.; SILVA-JUNIOR, M. C.; REZENDE, A. V.; NOGUEIRA, P. E.; WALTER, B. M. T.; SILVA, M. A.; ENCINAS, J. I. Comparação florística e fitossociológica do Cerrado nas Chapadas Pratinha e Veadeiros. In: LEITE, L.; SAITO, C. H. (Eds.). Contribuição ao conhecimento ecológico do Cerrado. Brasília: UnB, 1997. p. 6-11.

FIDELIS, A. T.; GODOY, S. A. P. Estrutura de um cerrado stricto sensu na gleba cerrado Pé-de-Gigante, Santa Rita do Passa Quatro, SP. Acta Botanica Brasilica, São Paulo, v. 17, n. 4, p. 531-539, 2003.

FIEDLER, N. C.; AZEVEDO, I. N. C.; REZENDE, A. V.; MEDEIROS, M. B.; VENTUROILI, F. Efeito de incêndios florestais na estrutura e composição florística de uma área de cerrado sensu stricto na fazenda Água Limpa-DF. Revista Árvore, Viçosa, v. 28, n. 1, p. 129-138, 2004.

HARIDASAN, M.; ARAÚJO, G. M. Aluminium acummulating species in two forest communities in the Cerrado region of central Brazil. Forestry Ecology Management, Amsterdam, v. 24, p. 15-26, 1988.

INSTITUTO NACIONAL DE METEOROLOGIA. INMET 1930-1990. Disponível em: <http//Lww.inmet.com.brì. Acesso: 28 out. 2008.

MARTINS, F. R. Estrutura de uma floresta mesófila. 2. ed. Campinas: Unicamp, 1993. 246 p.

MULlER-DOMBOIS, D. I.; ELLEMBERG, H. Aims and methods of vegetation ecology. New York: J. Wiley, 1974. 547 p.
NAIME, U. J. Solos da área mineira do polígono das secas. Informe Agropecuário, Belo Horizonte, v. 17, n. 181, p. 10-15, 1994.

NERI, A. V.; MEIRA-NETO, J. A. A.; SILVA, A. F.; MARTINS, S. V.; SAPORETTI-JUNIOR, A. W. Análise da estrutura de uma comunidade lenhosa em área de cerrado sensu stricto no município de Senador Modestino Gonçalves, Norte de Minas Gerais, Brasil. Revista Árvore, Viçosa, v. 31, n. 1, p. 123-134, 2007a.

NERI, A. V.; MEIRA-NETO, J. A. A.; SILVA, A. F.; MARTINS, S. V.; BATISTA, M. L. Composição florística de uma área de cerrado sensu stricto no município de Senador Modestino Gonçalves, Vale do Jequitinhonha (MG) e análise de similaridade florística de algumas áreas de cerrado em Minas Gerais. Revista Árvore, Viçosa, v. 31, n. 6, p. 1109-1119, 2007b.

PRIMACK, R. B.; RODRIGUES, E. Biologia da conservação. Londrina: [s.n.], 2001. 328 p.

RIBEIRO, J. F.; WALTER, B. M. T. Fitofisionomias do bioma Cerrado. In: SANO, S. M.; ALMEIDA, S. P. Cerrado: ambiente e flora. Planaltina: Embrapa-CPAC, 1998. p. 87-167.

RIZZINI, C. T. Tratado de fitogeografia do Brasil: aspectos ecológicos, sociológicos e florísticos. Rio de Janeiro: Âmbito Cultural, 1997. 747 p.

SCHILLING, A. C.; BATISTA, J. L. F. Curva de acumulação de espécies e suficiência amostral em florestas tropicais. Revista Brasileira de Botânica, São Paulo, v. 31, n. 1, p. 179-187, 2008.

SILVA, L. O.; COSTA, D. A.; FILHO, K. E. S.; FERREIRA, H. D.; BRANDÃO, D. Levantamento florístico e fitossociológico em duas áreas de cerrado sensu stricto no Parque estadual da Serra de Caldas Novas, Goiás. Acta Botanica Brasilica, São Paulo, v. 16, n. 1, p. 43-53, 2002.

SILVA-JÚNIOR, M. C. Fitossociologia e estrutura diamétrica na mata de galeria do Pitoco, na Reserva Ecológica do IBGE, DF. Cerne, Lavras, v. 11, n. 2, p. 147-158, 2005.

ZAR, J. H. Biostatistical analysis. 3. ed. New Jersey: Prentice Hall, 1996.

Cerne, Lavras, v. 16, n. 3, p. 267-281, jul./set. 2010 